\title{
Alimentación en el contexto familiar y escolar del niño con desnutrición de una zona rural
}

Food in the family and school context of the child with malnutrition in a rural area

\section{Alimentação no contexto familiar e escolar da criança com desnutrição em área rural}

Johana Yeraldine Barragán Piñin ${ }^{1}$, Katia Medali Torres Torres ${ }^{2}$, Angélica Soledad Vega Ramírez $^{3}$, Rosa Jeuna Díaz Manchay ${ }^{4}$, Flor De María Mogollón Torres ${ }^{5}$, Sonia Tejada Muñoz

${ }^{1}$ Licenciado en enfermería. Centro de Salud Paimas-Piura, Perú. Orcid: https://orcid.org/0000-00024040-9340 Correo electrónico: yohana_231@outlook.com

${ }^{2}$ Licenciado en enfermería. Instituto Superior Tecnológico Huarmaca-Piura, Perú. Orcid:

https://orcid.org/0000-0002-5560-7809 Correo electrónico: meda-9293@hotmail.com

${ }^{3}$ Magister en ciencias de enfermería. Especialista en salud familiar y comunitaria. Docente de la Universidad Católica Santo Toribio de Mogrovejo- Chiclayo, Perú. Orcid: https://orcid.org/0000-00017877-1436 Correo electrónico: avega@usat.edu.pe.

${ }^{4}$ Doctor en ciencias de enfermería. Especialista en salud familiar y comunitaria. Docente de la Universidad Católica Santo Toribio de Mogrovejo- Chiclayo, Perú. Orcid: https://orcid.org/0000-00022333-7963 Correo electrónico: rdiaz@usat.edu.pe.

${ }^{5}$ Magister en ciencias de enfermería. Especialista en neonatología. Docente de la Universidad Católica Santo Toribio de Mogrovejo- Chiclayo, Perú. Orcid: https://orcid.org/0000-0002-2605-546X Correo electrónico: fmogollón@usat.edu.pe.

${ }^{6}$ Doctor en ciencias de enfermería. Docente de la Universidad Nacional Toribio Rodríguez de Mendoza- Chachapoyas, Perú. Orcid: https://orcid.org/0000-0002-1181-8540 Correo electrónico:cieloceleste120@hotmail.com.

Cómo citar este artículo en edición digital: Barragán Piñin, J. Y., Torres Torres, K. M., Vega Ramírez, A. S., Díaz Manchay, R. J., Mogollón Torres, F. M. y Tejada Muñoz, S. (2020). Alimentación en el contexto familiar y escolar del niño con desnutrición de una zona rural. Cultura de los Cuidados

(Edición digital), 24 (56) Recuperado de http://dx.doi.org/10.14198/cuid.2020.56.12 Correspondencia: Facultad de Ciencias de la Salud, Universidad Católica Santo Toribio de Mogrovejo, Av. San Josemaría Escrivá de Balaguer Nº 855, CP: 14001. Chiclayo, Lambayeque. Perú. Correo electrónico de contacto: avega@usat.edu.pe

7Recibido:23/10/2019

Aceptado:10/02/2020

\section{ABSTRACT}

Introduction: Culture implies a repertoire of different customs, beliefs and practices that should be respected and considered when caring. Objective: To describe and understand the power in the family and school context of the child with malnutrition in a rural area. Methods: Qualitative study, with ethnographic design. The sample was represented by 19 informants: eleven mothers, one teacher, one nurse and six family members, selected for saturation and redundancy; data collected by participant observation, ethnographic interview and field diary and analyzed using content analysis. Results: a) Customs in the 
acquisition, storage, preparation and distribution of food, b) Beliefs, food deprivation and use of home remedies to diseases, c) Participation of mothers in nutritional status Program at school. Conclusions: Care of mothers preschool, they are influenced largely by the cultural component: Evidencing preferences give the best portion of food to the household head; low consumption of meat and vegetables; your way to heal disease by limiting the consumption of certain foods and inappropriate use of medicinal plants, a situation that could aggravate malnutrition.

Keywords: Culture, food, child, nursing, ethnography.

\section{RESUMEN}

Introducción: La cultura implica un repertorio distinto de costumbres, creencias y prácticas que deben respetarse y considerarse al cuidar. Objetivo: Describir y comprender la alimentación en el contexto familiar y escolar del niño con desnutrición de una zona rural. Métodos: Estudio cualitativo, con diseño etnográfico. La muestra estuvo representada por 19 informantes: once madres, un profesor, una enfermera y seis familiares, seleccionada por saturación y redundancia; los datos fueron recolectados por observación participante, entrevista etnográfica y diario de campo y analizados mediante análisis de contenido. Resultados: a) Costumbres en la adquisición, conservación, preparación y distribución de los alimentos, b) Creencias, privación de alimentos y uso de remedios caseros ante enfermedades, c) Participación de las madres en el Programa nutricional del estado en la escuela. Conclusiones: Los cuidados de las madres al preescolar, están influenciados en gran medida por el componente cultural: Evidenciándose preferencias en dar la mejor porción de comida al jefe del hogar; consumo escaso de carnes y verduras; su forma de sanar enfermedades limitando el consumo de ciertos alimentos y el uso inadecuado de plantas medicinales, situación que podría agravar la desnutrición.

Palabras clave: Cultura, alimentación, niño, enfermería, etnografía.

\section{RESUMO}

Introdução: Cultura implica um repertório diferente de costumes, crenças e práticas que devem ser respeitados e considerados ao cuidado. Objetivo: Descrever e compreender a alimentação no contexto familiar e escolar da criança com desnutrição em área rural. Métodos: Estudo qualitativo, com design etnográfico. A amostra foi representada por 19 entrevistados: once mães, um professor, um enfermeiro e seis membros da família, selecionados por dados de saturação e redundância; dados coletados por observação participante, entrevista etnográfica e diário de campo e analisados utilizando análise de conteúdo. Resultados: a) Customs na aquisição, armazenamento, preparação e distribuição de alimentos, b) Crenças, de privação alimentar e uso de remédios caseiros para doenças, c) Participação das mães no Programa estado nutricional na escola. Conclusões: Cuidado de mães pré-escola, eles são influenciados em grande parte pelo componente cultural: Evidência de preferência em dar a melhor porção de comida para o chefe de família; baixo consumo de carne e legumes; sua maneira de curar a doença, limitando o consumo de certos alimentos e uso inadequado de plantas medicinais, uma situação que pode agravar a desnutrição.

Palavras-chave: Cultura, comida, criança, enfermagem, etnografia.

\section{INTRODUCCIÓN}

La alimentación evoluciona con el tiempo, y en ella influyen muchos factores, como: los ingresos económicos, el costo de los alimentos (que afectarán la disponibilidad y asequibilidad de alimentos saludables), las preferencias y creencias individuales, las tradiciones culturales, y los factores geográficos y ambientales (incluido el cambio climático) (OMS, 2018). Mientras, el coste de la malnutrición es de unos 3,5 billones de dólares por año; hay 821 
Revista científica de la Asociación de Historia y Antropología de los Cuidados (Universidad de Alicante)

millones de personas que padecen hambre crónico; 151 millones de niños menores de cinco años tienen retraso del crecimiento (FAO, 2019). De este modo, la pobreza conlleva a una dieta insuficiente y por ende a la desnutrición y problemas de salud. La alimentación en las zonas rurales, por lo general se caracterizan por contener alimentos propios de la zona obtenidos por producción o por trueque en las ciudades próximas y muy pocos por compras gracias a un ingreso suficiente (Blanco, 2012).

Entre el 2000- 2011, Perú ha logrado disminuir sus tasas de desnutrición y de anemia; a pesar de ello, siguen siendo muy altas, lo que constituye un verdadero desafío para las políticas públicas (Sobrino et al, 2014); además, en la provincia de Lambayeque el mayor porcentaje, 27.3\% está en el distrito de Salas (ASIS, 2016). En 2016, unos 155 millones de niños menores de 5 años, tenían retraso en el crecimiento (CES, 2017). Ante esto, se deduce que la etapa preescolar es la más vulnerable a enfermedades, como la anemia y el retardo en el crecimiento físico. Por tanto, un niño desnutrido consecuente de una malnutrición durante esa etapa de su vida, es inerme a los efectos negativos de dicha condición; hay que considerar, además, que la alimentación depende de los padres, quienes pueden no tener los recursos económicos adecuados, sumando al bajo nivel de instrucción y los insuficientes conocimientos para llevar a cabo esta tarea de manera correcta, repercutiendo negativamente en la salud del niño.

Mendoza, (2016), establece que las creencias perjudiciales, como: el inicio precoz de la alimentación complementaria, antes de los 6 meses, consumir sólo 2 comidas principales al día en lugar de 3 y consumir alimentos disminuidos en frecuencia, consistencia y cantidad durante los procesos infecciosos, así como la eliminación de excretas al campo abierto en lugar de usar inodoro o letrina, son prácticas asociadas a la aparición de desnutrición. Por consiguiente, teniendo presente la pluriculturalidad que existe en Perú, el personal de salud debe acoplar sus conocimientos con las creencias de la población y así proporcionar cuidados de forma responsable y coherente con la cultura, los que serán orientadas hacia tres aspectos, como afirma (Leininger \& McFarland, 2006), el primero: preservar y/o mantener los cuidados culturales, segundo, adaptar y/o negociar estos cuidados $\mathrm{y}$ finalmente reorientarlos y/o reestructurarlos.

Por lo mencionado, es inevitable dejar de preocuparse por el cuidado en la alimentación, que brindan las madres de las zonas rurales, a los niños que padecen desnutrición. Es así que se desarrolló la investigación en el centro poblado "Sauce”, distrito de Salas, departamento de Lambayeque-Perú, donde se evidenció que las personas hacen un gran esfuerzo por 
obtener ingresos económicos, de las actividades productivas propias de la zona rural, como son la agricultura y la ganadería, para asegurar una de las necesidades básicas de sus miembros, la alimentación, aunque esta no sea la más adecuada; además denotan una variedad de creencias sobre el cuidado en la alimentación, mismas que repercuten en el estado de salud de la población y particularmente en la de los niños. El escenario fue elegido por ser uno de los distritos donde existe alta prevalencia de desnutrición en preescolares y por ser un lugar muy conocido en Perú por sus prácticas de la medicina tradicional.

En este marco de referencia, resultó importante develar las creencias y las prácticas alimentarias, ya que concepciones erróneas pueden condicionar hábitos alimentarios poco saludables, con repercusiones negativas a futuro; así mismo, resultó relevante examinar y valorar aquellas prácticas protectoras que poseen estas familias, y que pueden ser reforzadas. Es así que se realizó la investigación, con el objetivo de: describir y comprender la alimentación en el contexto familiar y escolar del niño con desnutrición de una zona rural.

\section{MÉTODO}

La investigación fue de tipo cualitativa, con abordaje etnográfico (Grove, Gray \& Burns, 2016; Do Prado, De Souza, Monticelli, Cometto \& Gómez, 2013, p.
253). Para lo cual, las investigadoras se involucraron directamente con los informantes, y además se observó el escenario cultural donde la madre interactuó según sus creencias y costumbres adquiridas culturalmente en la alimentación del niño con desnutrición. Los informantes fueron 11 madres de familia de la zona rural "Sauce”, con niños en edad preescolar con desnutrición, el cual se verificó en el carné de crecimiento y desarrollo. Además, se consideró 8 informantes: docente (1), enfermera (1) y familiares (6) con los que conviven los preescolares.

Los participantes en este estudio, fueron elegidos por muestreo no probabilístico, por conveniencia y ninguno abandonó el estudio una vez iniciado el mismo; sin embargo, cabe mencionar, que fue necesario regresar más de 4 veces, en la mayoría de los casos, para encontrar a las madres, debido a sus múltiples labores del hogar; en tanto que, con las docentes de la escuela, no se presentó ninguna dificultad en su participación. Las características resaltantes de las madres fueron: edades entre 19 y 62 años, con estado civil conviviente, grado de instrucción primaria o primaria incompleta, procedentes de zonas rurales (sierra de Lambayeque y de Piura); con respecto a la docente de la escuela de la zona, 32 años de edad, 5 años de labor como docente, de los cuales 2 en la localidad de Salas, procedente de Chiclayo y nunca recibió capacitación sobre alimentación en niños. La enfermera es una profesional que 
Revista científica de la Asociación de Historia y Antropología de los Cuidados (Universidad de Alicante)

labora en el establecimiento de salud del primer nivel de atención del sector, durante 6 horas, de lunes a viernes brindando cuidados preventivo promocionales; con respecto a los familiares, fueron una tía y cinco abuelas, quienes asumen el rol de madre cuando ellas se van a trabajar en labores de agricultura en sus chacras (granjas o propiedades).

Para asegurar la confidencialidad, se asignaron los siguientes códigos de identificación; para las madres: MADR1, MADR2...MADR11, para la enfermera ENF, para los familiares F01, F02...F06 y para el docente DOC. El tamaño de la muestra estuvo determinada por la técnica de saturación y redundancia, es decir, cuando la información de las entrevistas se repitió y no se encontraron datos nuevos. Como técnica de recolección de datos, se utilizó la observación participante y la entrevista etnográfica; como instrumentos, la guía de observación que se registró en el diario de campo y la guía de entrevista, cuyos datos fueron grabados en un grabador de voz. Cabe mencionar que dichos instrumentos, fueron elaborados por las investigadoras y validados por juicio de expertos y mediante un acercamiento al campo para verificar conceptos culturales, mismo que se realizó con 05 madres de familia del distrito de Mórrope, lugar con similares características del escenario donde se realizó la investigación, cuya participación permitió modificar algunos términos de las preguntas, a un lenguaje más sencillo y comprensible para los participantes.

A cada participante se le visitó 4 veces, por un promedio de cuatro horas en cada visita; la estrategia que se utilizó para captar a las madres fue acompañar al personal de salud (enfermera) en las visitas domiciliarias, solicitando la revisión del carné de control de crecimiento y desarrollo del niño, esto facilitó realizar la observación y la entrevista a las madres, interactuando con cada una de ellas, hasta ganarse su confianza, llegando a involucrarse en las actividades cotidianas relacionadas con el cuidado en la alimentación al preescolar con desnutrición; la entrevista fue en un tiempo promedio de 35 minutos, al finalizar las tareas cotidianas de las madres, procurando el mejor momento y libre de interrupciones. A la enfermera y docente se le aplicó la entrevista al finalizar su jornada laboral y tuvo una duración de 30 minutos en promedio.

Para el procesamiento de datos fue de manera manual, mediante el análisis temático (McCurdy, Sprandel \& Shandy, 2005) siguiendo cuatro etapas: a) Análisis del dominio: en el cual se analizó los datos referentes a situaciones sociales, identificando las costumbres obtenidas por medio de la observación participante y los discursos de las entrevistas, los cuales se analizaron para establecer los dominios culturales, b) Análisis taxonómico: donde se 
construyeron relaciones semánticas entre todos los términos incluidos en los dominios. Una taxonomía revela la existencia de subconjuntos de términos relacionados en un todo, c) Análisis componencial: es el análisis por el cual se buscó componentes de significado, actividad reflexiva que permitió comprender el significado de un término en la medida que difiere de los demás y d) Análisis de temas: que consistió en describir la cultura observada señalando sus características generales y específicas. El objetivo fue identificar los elementos cognitivos que constituyen una cultura, lo que sus integrantes creen que es y reconocen como real. En toda la investigación, se cumplieron con los principios de la Ética Personalista de Sgreccia, (2009). Los participantes no fueron expuestos a experimentación, ni sufrieron ningún daño físico, ni emocional, se protegió su identidad con códigos y su participación fue voluntaria, previa firma del consentimiento informado, además de contar con la aprobación del Comité de Ética de la Facultad de Medicina de la Universidad Católica Santo Toribio de Mogrovejo.

Asimismo, se cumplió con los criterios de rigor científico de Denzin \& Lincoln, (2012). Por tanto, se garantiza la credibilidad mediante la transparencia y fidelidad en relación con los datos encontrados, los cuales fueron recolectados durante un periodo de tiempo amplio, y fueron construidos e interpretados de forma cuidadosa y ejemplificada a través de las respuestas de los participantes. Igualmente, la credibilidad se asegura mediante la triangulación de la información obtenida a través de múltiples fuentes (entrevistas individuales y observaciones); diferentes contextos (casa, institución de salud y escuela), y los diferentes actores (madres, enfermera, docente $y$ familiares), que ampliaron la visión sobre el fenómeno de la investigación. También, con la devolución de la información a los actores participantes se aseguró la credibilidad, pues permitió corroborar lo establecido por ellos, lo que permitió agregar algunos aspectos poco abordados en las entrevistas. La auditabilidad, se garantizó mediante la existencia de grabaciones de las entrevistas realizadas, los registros de las entrevistas, la transcripción fiel de los diálogos, las observaciones y la descripción de los contextos sociales donde se llevó la investigación. La transferibilidad o aplicabilidad se aseguró al presentar los resultados que identifican al grupo de estudio en su contexto social.

\section{RESULTADOS}

El análisis de los textos producidos por las fuentes listadas en la interacción con los diferentes actores permitió construir tres temas culturales:

\section{a) Costumbres en la adquisición,} conservación, preparación y distribución de los alimentos

Las costumbres de las madres en la alimentación del niño con desnutrición, 
Revista científica de la Asociación de Historia y Antropología de los Cuidados (Universidad de Alicante)

envuelven un proceso complejo de toma de decisiones en cuanto a la adquisición, conservación, variedad, preparación, cantidad, de los alimentos, así como horarios en la dispensación de la dieta diaria, entre otros aspectos, mismos que se transmiten de generación en generación. Una influencia para la ingesta inadecuada de nutrientes es la insolvencia económica para comprar alimentos con alto contenido de proteínas y otros que se requiere para una dieta balanceada, como se denota en la siguiente narrativa: "Mis animalitos los vendo para tener dinero y poder comprar arroz, fideos o verduras”(MADR 11), “cocinamos lo que hay, este año ha sido seco no sé ha sembrado por falta de agua....solo los sábados compro zapallo, apio y repollo, para toda mi semana...la enfermera dice que le dé pollo con fideo cuando se enferma, pero no hay ni plata para comprar” (MADR 3, 4).

La técnica más popular que usan para conservar sus alimentos es el "secado de las carnes”, costumbre primitiva usada por muchas culturas, en diferentes épocas $\mathrm{y}$ partes del mundo, a la falta de tecnología, de conservantes artificiales y herramientas de refrigeración necesarias. Se observó que las madres "tienen como costumbre secar sus carnes en su cordel de tender ropa, sajadas de carne de pavo expuestas al sol (Diario de campo -hogar de las madres). Esto se corrobora: "nuestras carnecitas, que rara vez compramos, lo colgamos en el tendedor para que seque y no se malogre” (MADR 2).
La mayoría de las viviendas ubicadas en la localidad son de material de adobe con barro, cocinan con leña. Además, algunas familias tienen mesas de madera con bancas alrededor, o una tabla sobre ladrillos (que cumple las veces de una mesa) y algunos ladrillos como asientos (en lugar de sillas), cuelgan los utensilios de cocina en un alambre en la pared. "Al momento de preparar los alimentos, las madres tienen los cabellos sueltos, las ollas presentan hollín en el interior y exterior, la mesa o superficies usadas para picar los alimentos, como las verduras, presentan restos de comida, residuos de desechos alimentarios (cáscaras, hojas, envolturas) ... conviven juntos con los animales que crían (gallinas, patos, perros, gatos, cuyes en jaula), pero también crían animales en la intemperie, como: ovejas, chanchos en su mayoría. (Diario de campo -hogar de las madres).

En cuanto a la dieta familiar, se evidenció que esta no es balanceada, por lo común es en base a carbohidratos: “comemos lentejas, alverjas, fideo, papa y su arroz como siempre, porque no venden verduras. La harina de trigo lo usamos para hacer tortas fritas y el trigo pelado para sopas o como menestra” (MADR 4), “En los desayunos se suele cocinar papas o plátanos con un huevo frito o queso; en el almuerzo cocinamos arroz con menestra verde, sopa de fideo con algunas verduras $y$ pollo cuando tenemos, dos veces al mes comemos pato o gallina que los criamos en 
Cultura de los Cuidados

el corral” (MADR 2, 3). "La comida del almuerzo lo guardo para la noche lo que sobra” (MADR 11), “A mis hijos les doy de comer a su hora, comemos a la una o medio día, y cenamos a las siete y a las nueve los niños se van a dormir" (MADR 9).

Por otro lado, hay elementos culturales arraigados que guían la distribución o repartición de la dieta en las familias, se da mayor importancia al padre, la madre o al pariente, que aporta económicamente, que a la necesidad nutricional de los niños. Así lo demuestra el siguiente discurso: "almorzamos a eso de la una, si esta mi esposo le sirvo a el primero su buen plato, porque es el jefe, luego mis hijitos y yo, casi siempre comemos en familia" (MADR 3, 6, 10).

Estas situaciones, evidencian claramente que la desnutrición presente en los preescolares podría estar muy relacionado con las prácticas culturales de las madres, quienes además de no acudir a la institución de salud para la asistencia sanitaria, tienen costumbres que agudizan la desnutrición. Esto se corrobora con el discurso de la enfermera: "la desnutrición de estos niños es crónica - mostrando carné de control de crecimiento- el niño pierde peso, se enferma frecuentemente, empieza a perder apetito y vivacidad...Desde el punto de vista funcional, se afectan sus capacidades, tanto intelectuales, como de atención y las madres no recurren a nosotros cuando sus niños se enferman, $y$, es más, no acuden a los controles de sus niños" (ENF). Siendo necesario un mayor seguimiento desplazándose al hogar de los niños desnutridos, considerando los recursos con los que cuentan en casa y educando de manera personalizada según la necesidad de cada familia.

\section{b) Creencias, privación de alimentos $y$ uso de remedios caseros ante enfermedades}

Las madres del estudio tienen creencias que podrían empeorar la desnutrición de sus niños, pues los privan de ciertos alimentos para sanar enfermedades. Al respecto, evitan el pescado, carne de pato o papa cuando el niño tiene leishmaniasis; evitan alimentos fríos y dulces, como: plátano o naranja, ante la gripe; frotan con sal o huevo el cuerpo de sus hijos para limpiarlos del susto, entre otros: "Cuando mi hijo enferma de uta (leishmaniasis, no le damos huevo, queso, carne fresca, papa, pescado, porque hacen que se madure y reviente la herida y el pescado infecta la herida” (MADR2, MADR8), "cuando se enferma de susto, le paso sal o alumbre por su cuerpo" (MADR 2).

Asimismo, tienen la costumbre de frotar el cuerpo con el cuy, muy conocido en medicina tradicional. Así lo explican las madres: "Cuando mi niña se enferma y no quiere comer le paso cuy por todo su cuerpito y con eso le pasa y ya tiene hambre" (MADR 3, 7 y 10).

Por otro lado, las madres también usan plantas medicinales propias de la zona como la flor de overo, achicoria, manzanilla, 
Revista científica de la Asociación de Historia y Antropología de los Cuidados (Universidad de Alicante)

romero, etc. para sanar algunas jugar, ya que no todos llevan cuaderno, en su enfermedades; ellas consideran que todos estos conocimientos culturales se deben aplicar porque según los usos, han dado resultados positivos en beneficio de la salud de sus hijos. Así manifiestan: "la infusión de achicoria entre comidas estimula el apetito en nuestros hijos” (MADR9), “cuando mi hija tiene fiebre, mi mamá le da agua de flor de overo con achicoria y cuando le duele su barriga, manzanilla” (MADR1). Además, las investigadoras observaron que las madres “acostumbran a secar y guardar grasa de gallina y usarlas como remedio para el estreñimiento e infecciones respiratorias” (Diario de campo- hogares de las madres).

\section{c) Participación de las madres en el} Programa nutricional del estado en la escuela.

Los niños preescolares del estudio asisten a un PRONOEI, es un programa no escolarizado infantil, ejecutado a nivel nacional en zonas de extrema pobreza y de mayor índice de desnutrición crónica infantil, forman parte de una política de estado que busca ampliar la cobertura educativa $y$ en reconocimiento de la diversidad cultural del Perú, cuentan con un comedor escolar con el objetivo de contribuir al mejorar el rendimiento escolar a través de la complementación alimentaria. Se dictan clases a los niños de 2 a 5 años, el ambiente es muy pequeño, solo cuenta con dos 2 mesas medianas para que ellos puedan mayoría únicamente llevan su plato para la comida que van a recibir.

Es evidente, que la madre por desconocimiento o insolvencia económica no provee adecuadamente los nutrientes que requiere el menor, tanto en casa, como en su lonchera para la escuela: "en la escuela donde se dictan clases a los niños de 2 a 5 años, la madre se encarga de cocinar para todos los niños, utiliza los alimentos que le da el estado por el programa de salud: avena, quinua y leche en polvo" (Diario de campo-escuela). Los familiares manifestaron: "a veces le mando huevito pasado, pero mayormente no, porque en la escuela, de lunes a viernes, les dan desayuno y almuerzo, come papitas o yucas fritas con arroz y lentejita” (F03), "yo le envío a mi nieto en su lonchera plátano y huevo frito con café o yuca con queso" (F06). "Señorita, yo no le pongo lonchera, porque allá les dan desayuno y almuerzo, pero su profesor ya debe ver todo eso" (MADR 5, $10)$.

Lo antes mencionado, se corrobora también con los discursos vertidos por el docente: "una madre es la que se encarga de cocinar para todos y se van rotando entre todas; los alimentos vienen del estado y les entregan cada mes o 3 meses, los niños no traen lonchera para su refrigerio porque aquí reciben desayuno y almuerzo de lunes a viernes; pero existen unos 3 niños que en su excepción llevan lonchera con contenido de bajo valor nutricional, hecho que se 
evidenciaba por la presencia de galletas, frituras, café o té” (DOC). Por ende, las investigadoras consideran que la persona que educa a los niños y a la vez está involucrado (a) en la supervisión de la alimentación de los niños los días que enseña, no se encuentran capacitados en su totalidad, les falta mayor asesoramiento, por lo cual el estado y el centro de salud debe preocuparse, por esta situación.

\section{DISCUSIÓN}

El aporte de esta investigación, para enfermería, radica en que al estudiar la alimentación del niño con desnutrición desde lugares habituales como son el domicilio, la escuela y la institución de salud, contribuyen a entender el cuidado émic, cuidado genérico propio de los seres humanos y comunidades, sus prácticas, costumbres, creencias y modos tradicionales para proporcionar actos de ayuda, asistencia y apoyo hacia sí mismos y a otros para lograr el bienestar, mejorar condiciones humanas y brindar un cuidado culturalmente congruente (Leininger \& McFarland, 2006). La cultura influye definitivamente en las creencias, conocimientos percibidos, prácticas, valores y modos de vida (Leininger \& McFarland, 2006) consecuente con los resultados de este estudio; el cuidado émic de las madres en la alimentación de su hijo preescolar con desnutrición en una zona rural, resultante de factores de la estructura social representados por Leininger en el modelo del sol naciente (Leininger \& McFarland, 2006), son factores culturales de su entorno, que inciden en sus modos de cuidar. Esto es evidente, cuando se describe que, al enfermar el niño, lo privan del consumo de carne de pato, pescado, naranja y papa o al practicar el uso de plantas o hierbas, para sanar algunas enfermedades propias de la zona, como la flor de overo, llantén, manzanilla, romero, etc.

Las costumbres que tienen las familias que participaron en el estudio, para adquirir, conservar, preparar y distribuir la dieta familiar; resultan inadecuadas para nutrir al niño en edad preescolar que padece desnutrición, a consecuencia de muchos factores, como: los bajos ingresos económicos que los obtienen básicamente de la agricultura o de la venta de sus aves de corral. Por lo que, la dieta es a base de carbohidratos, pues cultivan según el clima de la zona: papas, ollucos, trigo. Esto se corrobora, con algunos estudios realizados en el Perú, sobre las costumbres y prácticas relacionadas a la alimentación infantil, identificaron aspectos comunes, como la interrupción de la lactancia materna antes del $6^{\circ}$ mes de vida, e incluso desde el nacimiento optan por alimentar a sus hijos con leche de vaca, gelatina, infusiones; exclusión de alimentos propios de la zona como quinua, quiwicha, oca, choclo y el rechazo de los micronutrientes llamados “chispitas" (Villegas \& Arriola, 2016; Campos \& Castillo, 2014; Bustamante \& Gordillo, 2017). 
Revista científica de la Asociación de Historia y Antropología de los Cuidados (Universidad de Alicante)

Algo que resulta paradójico es que crían aves de corral y cuyes, pero los venden para que puedan comprar alimentos, como: fideos, arroz, azúcar, esto debido a que estos alimentos "llenan y dan energía", que no cubren los requerimientos nutricionales proteicos que necesitan sobre todo los niños con desnutrición. Contrariamente a lo que concluye Ger, (2018), en una zona rural de Ecuador, donde los escolares si tienen acceso a alimentos proteicos de alto valor biológico ya que en los hogares crían animales, producen leche y huevos, que sirven para el consumo propio y la población que no crían, acceden a estos alimentos mediante la compra frecuente; y el estado nutricional de la mayoría de los escolares es normal; sin embargo, existen porcentajes de sobrealimentación, como: el sobrepeso (33\%) y obesidad (12\%), debido a que les gusta más consumir alimentos fuentes de carbohidratos, como, el arroz, pan, tallarín y papas fritas.

En este estudio se evidencia, una inadecuada ingesta de nutrientes en el niño, influenciado por las costumbres familiares, basado en el consumo excesivo de carbohidratos, toda vez que consumen bastante papa, lentejas, quinua, etc., y pobre en proteínas y grasas, debido a la insolvencia económica para adquirir otros alimentos, unido a la desinformación sobre una dieta balanceada, lo que hace que, en la cena, consuman lo que queda del almuerzo. Al respecto, Teijón, Blanco, Olmo, Posada, Teijón \& Villarino
(2017), afirman que los alimentos ricos en proteínas, son: el huevo, leche, carne y pescado, y aportan una cantidad óptima para cubrir necesidades del organismo; pero, una correcta nutrición se basa en una dieta apropiada, que aporte los nutrientes necesarios (carbohidratos, proteínas, grasas, vitaminas y minerales); las necesidades energéticas de una persona varían entre 1000 y $4000 \mathrm{kcal} /$ día dependiendo de su edad, sexo y actividad. Los niños pequeños crecen rápidamente, sus necesidades energéticas y nutritivas son relativamente altas para su tamaño corporal (Gonzáles, 2017).

Por otro lado, en este estudio se encontró, que la alimentación es a base de productos propios de su zona, resaltando la preferencia de dar más cantidad de comida al padre por ser la persona que sustenta la familia. En este sentido, la promoción de las comidas en familia y compartidas se considera ya una estrategia de salud pública que requiere la puesta en marcha de acciones específicas de prevención dirigidas a incrementar la frecuencia y a mejorar la calidad (tipos de alimentos servidos, tamaño de las porciones, ambiente en el que se sirve) de las comidas familiares (Blake, 2018). También tienen la costumbre de secar las carnes para conservarlas, en cambio en la selva peruana la tierra es usada como una especie de «refrigerador», que la usan para conservar algunos productos, cavando y guardando en ella principalmente yuca, 
plátano y camote (Rumrrill, Arnold, Chávez, Godoy \& Ancapan, 2015)

Un aspecto importante a rescatar en este estudio, es que comen en familia y se brindan a la hora adecuada, los alimentos al niño. Desde el punto de vista nutricional comer en familia frecuentemente se asocia con el seguimiento de dietas más equilibradas y de mayor calidad y ciertas ventajas en el estado nutricional y de salud de las personas que lo frecuentan, especialmente niños más vulnerables, como preescolares con desnutrición (Skafida, 2013; Fulkerson, J. A., Larson, N., Horning, M. \& Neumark-Sztainer, D., 2014). Las madres andinas peruanas, ciertamente evidencian un sistema alimentario que se compone, en tanto sistema simbólico, de normas, creencias, significados y asociaciones sobre qué es comida, quién o quiénes consumen qué, quién prepara y como preparan. Estos resultados concuerdan con lo encontrado en México (Contreras \& Arnaíz, 2005).

Con respecto al cuidado en la cocina, se evidenció que existe falta de higiene para la preparación y conservación de los alimentos, escaso lavado de manos antes de comer, hay presencia de moscas por los desperdicios, lo que puede conllevar a que los niños padezcan de enfermedades infectocontagiosas. Contrario a la seguridad alimentaria en el hogar, que es el acceso seguro y en todo momento de los hogares a alimentos suficientes en cantidad y calidad para que todos sus miembros puedan desarrollar una vida sana, productiva y activa (Ger, 2018) y a la manipulación, almacenamiento y transporte seguros, para lo cual deberán establecerse procedimientos sencillos para eliminar de manera higiénica los desechos, y proteger los alimentos (Armendáriz, 2012).

En este estudio se demuestra que existen creencias inadecuadas en cuanto a la alimentación del niño cuando enferma, sobre todo la privación de ciertos alimentos como la carne de pato, el pescado, la naranja, la papa; causando un aporte inadecuado de nutrientes mientras dura el proceso de la enfermedad, siendo lo correcto recibir más proteínas para la recuperación y funcionamiento del organismo, lo cual exacerba la desnutrición infantil. Estas prácticas, requieren ser modificadas progresivamente, con argumentos convincentes para las madres, puesto que son perjudiciales para la salud del niño, en tal sentido, el profesional de enfermería precisa dar pautas de manera repetitiva y de alta intensidad, para lograr una práctica saludable (Pender, Murdaugh \& Parson, 2010), no solo orientando a la madre, en cuanto a la selección de los alimentos, sino en los aportes y beneficios de estos para los preescolares.

Por otro lado, también usan plantas o hierbas para sanar algunas enfermedades propias de la zona, como la flor de overo, llantén, manzanilla, romero, etc. todos estos conocimientos culturales ellas consideran que se deben tener en cuenta porque según 
Revista científica de la Asociación de Historia y Antropología de los Cuidados (Universidad de Alicante)

los usos han dado resultados positivos en beneficio de la salud de sus hijos. Esto coincide con Lillo \& Vizcaya, (2012), cuando afirman que, para los integrantes de las distintas culturas, los alimentos son interpretados como una amplia gama de símbolos, expresando y creando relaciones entre hombres, entre hombre y mujer, entre el propio hombre y los dioses, con la naturaleza, etc. Además, es común utilizar ritos mágicos conocidos como curanderismo, brujería o chamanismo; aquí realizan un ritual muy popular llamado "la limpia del cuy” que consiste en pasar el cuy por todo el cuerpo del enfermo, acompañado de rezos, dicho animal al final tiene que ser sacrificado para que puedan obsérvalo y detectar que parte o miembro tiene afectada la persona y que es lo que produce dicho mal. $\mathrm{Al}$ respecto cuenta la leyenda que los incas "leían” estrategias de guerra en las entrañas de los cuyes. Hoy en día, en cambio, se examinan posibles enfermedades de la gente utilizando este animal: la llamada "soba del cuy” es una antigua tradición de sanación de la Sierra Andina que practican los sabios de los pueblos (Gonzáles, 2017).

Otro aspecto importante, a considerar es que las madres no preparan lonchera para que lleven sus hijos preescolares, debido a que ellas se turnan cada semana para que en el PRONOEI preparen el desayuno y el almuerzo con los recursos del estado, cabe profundizar en otro estudio el valor nutritivo de estas dietas.
Qali Warma juega un rol importante en la nutrición escolar garantizando el servicio alimentario de calidad durante todos los días del año escolar y en cogestión con los padres, las madres y los profesores. De esta manera, el programa alinea sus esfuerzos con los Objetivos de Desarrollo Sostenible en la erradicación de la pobreza y la desigualdad, sin dejar a nadie atrás (Gonzales, 2019). Frente a ello, se crea la necesidad significativa del trabajo de la enfermería en instituciones educativas. Esta situación, obliga a que los profesionales de enfermería apliquen los principios que propone Leininger (Leininger \& McFarland, 2006), en su teoría del sol naciente, en la que se da valor a las prácticas y saberes culturales de la madre, de modo que se respete las practicas no nocivas y se trate de modificar paulatinamente, aquellas que sí lo son, buscando así un punto de equilibrio de ambas culturas: el de la madre y el de la enfermera. Por tanto, precisan negociar estas prácticas alimenticias con las madres, a fin de llegar a un acuerdo y mejorar la alimentación del preescolar.

\section{CONCLUSIONES}

Los cuidados de las madres a los preescolares están influenciados, en gran medida, por el componente cultural, dado que, las prácticas y creencias emergen de esta dimensión. Los factores socioculturales que delimitan de manera importante este cuidado corresponden a las creencias, 
valores, insolvencia económica, parentesco y estilos de vida. Las preferencias en dar la mejor porción de comida al padre; las carnes y verduras consumidas escasamente, su forma de sanar enfermedades privando el consumo de ciertos alimentos y el uso inadecuado de plantas medicinales podrían empeorar la desnutrición crónica infantil. Además, la falta de seguimiento a los programas del Estado trae consigo que la preparación de los alimentos no sea la adecuada, puesto que son las madres las que preparan los alimentos, sin la debida supervisión. Siendo imperativo el trabajo de la enfermería en instituciones educativas.

En esta investigación se ha tomado como referente teórico, los conceptos de Leininger (Leininger \& McFarland, 2006) de su Teoría del Sol Naciente, al implementar su propuesta, permitió realizar el contraste entre las dimensiones socioculturales que influyen en el cuidado de la madre en la alimentación del preescolar. Este modelo destaca la importancia de identificar las influencias que, sobre el cuidado y la cultura, ejercen algunos factores como el educativo y económico, las creencias y los estilos de vida, así como factores tecnológicos, sociales y de parentesco, políticos, religiosos $\mathrm{y}$ filosóficos; por tanto se puede inferir que existe una relación entre la desnutrición crónica de los preescolares y las prácticas de cuidado cultural de la madre en el contexto familiar y escolar demostrando su aplicabilidad en esta investigación.
Se sugiere hacer visitas continuas a las familias de zonas rurales para educar mediante demostraciones las prácticas correctas de almacenamiento y conservación de los alimentos, el lavado de manos, la combinación y preparación de las comidas. Además, se necesitan políticas públicas y acciones intersectoriales y coordinadas tanto en educación, agricultura, trabajo, vivienda, agua y saneamiento, entre otras.

Conflicto de intereses: Los autores declaran no tener conflictos de intereses.

Financiamiento: Ninguno.

\section{BIBLIOGRAFÍA}

Análisis de la Situación de Salud (ASIS). (2016). Perfil Socio-Demográfico, de Morbilidad y

Mortalidad, Prioridades y Respuesta Social. Gerencia Regional de Salud Lambayeque Perú.

Armendáriz, J.L. (2012). Seguridad e higiene en la manipulación de alimentos. 2 ed. Madrid: Paraninfo.

Blake, J. (2018). Dedicar tiempo a las comidas familiares: influencias de los padres, entornos de comidas en el hogar, barreras y factores de protección. Rev Fisiología y Comportamiento, 193(B),248251. Recuperado de https://doi.org/10.1016/j.physbeh.2018.03.0 35

Blanco, T. (2012). Alimentación y Nutrición. Lima, Perú: Universidad Peruana de Ciencias Aplicadas (UPC).

Bustamante, J., Gordillo, M. (2017). Cuidado de la madre andina al lactante menor desde una perspectiva intercultural. 
Revista científica de la Asociación de Historia y Antropología de los Cuidados (Universidad de Alicante)

(Tesis de licenciatura). Chiclayo. biológico en niños escolares de la Escuela Universidad Católica Santo Toribio de Mogrovejo. Lambayeque - Perú.

Campos, M. \& Castillo, J. (2014). Cuidado cultural de la madre al neonato en el caserío de Marayhuaca, Ferreñafe. (Tesis de Licenciatura). Facultad de Medicina Humana. Escuela de Enfermería. Universidad Católica Santo Toribio de Mogrovejo: Chiclayo - Lambayeque. Perú.

Consejo económico y social (CES). (2017). Progresos en el logro de los objetivos del desarrollo del milenio. [Internet]. Recuperado de http://www.un.org/en/ga/search/view_doc.a sp?symbol=E/2017/66\&referer=http://www .un.org/es/documents/index.html\&Lang=S

Contreras, J., \& Gracia, M. (2008). Preferencias y consumos alimentarios: entre el placer, la conveniencia y la salud. En Díaz, C. \& Gómez, C. (Ed.), Alimentación, consumo y salud. Barcelona: La Caixa.

Denzin, N. \& Lincoln, Y. (2012). Manual de la investigación Cualitativa: El campo de la Investigación cualitativa. 3a ed. Vol 1. Barcelona: Gedisa.

Do Prado, M., De Souza, M., Monticelli, M., Cometto, M. \& Gómez, P. (2013). Investigación cualitativa en enfermería. Metodología y didáctica. Washington DC: Organización Panamericana de la Salud.

Fulkerson, J. A., Larson, N., Horning, M. \& Neumark-Sztainer, D., (2014). A review of associations between family or shared Meal frequency and dietary and weight status outcomes across the life span. Journal of Nutrition Education and behavior, 46(1), 219.

Ger, H.J. (2018). Estado Nutricional y acceso a alimentos proteicos de alto valor

Esperanza. (Tesis de Licenciatura). Ibarra -

Ecuador. Recuperado de

http://repositorio.utn.edu.ec/bitstream/1234 56789/8802/1/06\%20NUT\%20262\%20TR ABAJO\%20DE\%20GRADO.pdf

Gonzales, M. (2019). Programa de las Naciones Unidas para el Desarrollo y la Iniciativa de Pobreza y Desarrollo Humano de la Universidad de Oxford-PNUD Perú // Fotos: Bruno Cámara Rojo / PNUD Perú Qali Warma / PNUD Perú. Recuperado de https://pnudperu.exposure.co/alimentacionque-derrota-la-desigualdad.

González, I. (2017). Comida de rico, comida de pobre. Evolución de los hábitos alimentarios (Siglo $X X)$. España: Universidad de Sevilla, Secretariado de Publicaciones.

Grove, S., Gray, J. \& Burns, N. (2016). Investigación en enfermería. Desarrollo de la práctica enfermera basada en la evidencia. $6^{\text {a }}$ ed. Barcelona: Elsevier.

Leininger, M. \& McFarland, M. (2006). Culture Care Diversity and Universality: A Worldwide Nursing Theory. 2nd ed. Sudbury, MA: Jones and Bartlett.

Lillo, M. \& Vizcaya, M. (2012). Origen y desarrollo de los hábitos y costumbres alimentarias como recurso sociocultural del ser humano: una aproximación a la historia y antropología de los cuidados en la alimentación. Cultura de los cuidados, 0(11), 61-65. Recuperado de https://doi.org/10.14198/cuid.2002.11.11.

McCurdy, D., Spradley, J. \& Shandy., D. (2005). The cultural experience: Ethnography in Complex Society. $2^{\text {a }}$ ed. Estados Unidos de América: Waveland Pr Inc. 
Cultura de los Cuidados

Mendoza, G. (2017). Creencias y prácticas alimentarias e higiénicas en madres de niños menores de 5 años en el distrito de Anta, Áncash, Perú. Aporte Santiaguino, 9(2), 339-352. Recuperado de http://dx.doi.org/10.32911/as.2016.v9.n2.20 6.

Organización de las Naciones Unidas para la alimentación y la Agricultura (FAO). (2019). Nutrición. [Internet]. Recuperado de http://www.fao.org/nutrition/es/

Organización Mundial de la Salud (OMS). (2018). Alimentación sana. [Internet]. Recuperado

https://www.who.int/es/news-room/factsheets/detail/healthy-diet

Rumrrill, R., Arnold, I.Z., Chávez, F., Godoy, M. \& Ancapan, J. H. (2015). Sistemas alimentarios tradicionales de los pueblos indígenas de Abya Yala. FONDO INDÍGENA / Fondo para el Desarrollo de los Pueblos Indígenas de América Latina y El Caribe. [Internet]. Recuperado de http://www.fao.org/3/a-i4669s.pdf

Sgreccia, E. (2009). Manual de Bioética: La bioética y sus principios. 5ta ed. Madrid: Talisio.

Skafida, V. (2013). The family meal panacea: exploring how different aspects of family meal occurrence, meal habits and meal enjoyment relate to young children's diets. Sociol Health Illn. 35(6), 906-23. Recuperado de https://www.ncbi.nlm.nih.gov/pubmed/235 51143

Sobrino, M., Gutiérrez, C., Cunha, A.J., Dávila, M. \& Alarcón, J. (2014). Desnutrición infantil en menores de cinco años en Perú: Tendencias y factores determinantes. Rev Panam Salud Pública. 35(2):104-12. Recuperado de https://scielosp.org/pdf/rpsp/2014.v35n2/10 4-112/es

Teijón, J., Blanco, M., Olmo, R., Posada, P., Teijón, C. \& Villarino, A. (2017). Fundamentos de Bioquímica Metabólica. Madrid, España: Tébar Flores.

Villegas, E. \& Arriola, M. (2016). Conocimientos y Prácticas sobre Lactancia Materna Exclusiva en Madres Primíparas que acuden al Servicio de Crecimiento y Desarrollo del Hospital Naylamp. Acc Cietna. 4(1), 30.

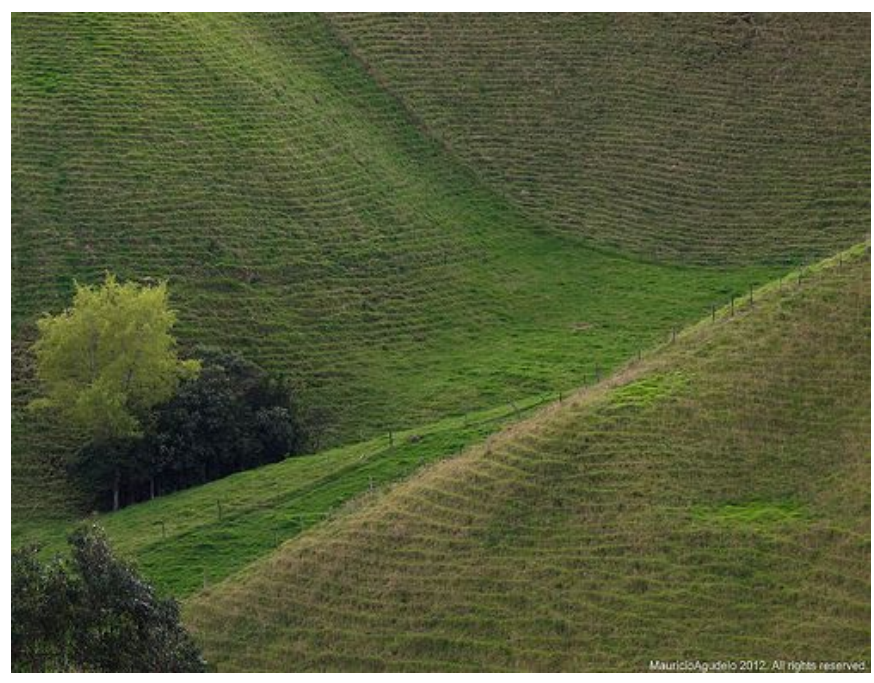

Fuente: https://www.pinterest.es/pin/135108057547135335/ 